\title{
Higher Order Generalized Impedance Boundary Conditions in Electromagnetic Scattering Problems
}

\author{
M. Duruflé, H. Haddar and P. Joly \\ INRIA-Rocquencourt, Domaine de Voluceau, BP 105, F-78153 Le Chesnay Cédex
}

\begin{abstract}
Résumé
Nous effectuons une revue succincte de l'obtention et l'utilisation des conditions d'impédances généralisées (GIBC) dans le cas de revêtements diélectriques minces et dans le cas d'objets fortement conducteurs. Nous nous plaçons dans le cadre des probèmes de diffraction d'ondes électromagnétiques en régime harmonique. Nous testons numériquement la validité et la précision de ces conditions aux limites pour le cas de forte conductivité. En présence de géométries comportant des coins, nous proposons un traitement numérique astucieux afin de garder la même précision que dans le cas de géométries régulières.
\end{abstract}

\begin{abstract}
We briefly review the use and the derivation of Generalized Impedance Boundary Conditions (GIBC) in the case of thin dielectric coatings and in the case of strongly absorbing media, within the context of electromagnetic scattering problem at a fixed frequency. We then numerically test the validity and accuracy of these boundary conditions in the case of high absorption. A numerical treatment of the corner singularity is proposed to recover the accuracy of the GIBC for singular geometries.
\end{abstract}

\section{Introduction}

The use of Generalized Impedance Boundary Conditions $(G I B C)$ in the mathematical modeling of wave propagation phenomena has gained a wide attention in recent decades (see for instance [19]). It is applied in electromagnetism to scattering problems from obstacles that are partially or totally penetrable. The general idea is to replace the use of an "exact model" inside (the penetrable part of) the obstacle by approximate boundary conditions (also called equivalent or effective conditions) in order to reduce the cost of numerical computations.

In this article, we shall focus on two types of electromagnetic time harmonic scattering problems involving the presence of a small length scale. The advantage of the approximate models is to avoid the use of computational meshes constrained by this small scale.

1. The scattering of electromagnetic waves by perfectly conducting obstacles coated with a thin layer of dielectric material. Due to the small thickness of the coating (typically with respect to the wavelength), the effect of the layer on the exterior field is, as a first approximation, local (see for instance, $[19,11,8,4]$, [16] for the high frequency limit, [1, 2] for periodic coatings and $[13,12]$ for an extension to the time dependent problem).

2. The scattering of electromagnetic waves by highly conducting materials. In such a case, it is the well-known skin effect that creates a "thin layer" phenomenon. The high conductivity limitates the penetration of the wave to a boundary layer whose depth is inversely proportional to the square root of its magnitude. Then, here again, the effect of the obstacle is, 


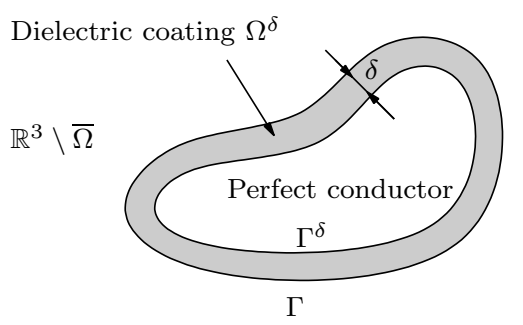

Figure 1: Thin layer configuration

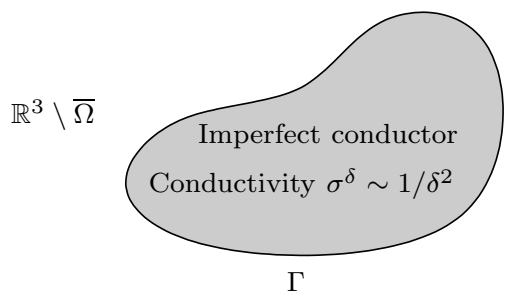

Figure 2: Configuration of highly conducting material

as a first approximation, local. The research on effective boundary conditions for highly absorbing obstacles began with Leontovich [5], then was pursued by Rytov [18] (see also [19]). More recent investigations, using different approaches, can be found in [7, 16].

This article is at the same time a review and a continuation of previous works $[12,14,15]$ that provides a numerical justification of the use of GIBCs. After having introduced our two model problems in section 2, we give and compare in section 3 the expressions of the GIBCs for each problem and for various orders of approximation (the notion of order is defined in section 2). These conditions require smoothness assumptions on the geometry of the obstacle. In section 4, we present the principles of the scaled asymptotic-expansions method, that enable us to derive the GIBCs. Section 5 is devoted to the numerical validation of the obtained GIBCs in the case of the high conductivity. From the numerical point of view these boundary conditions have the advantage of being variational (section 5.1) and are therefore suitable for finite elements type approximations (see also [21] for an integral equation approach). In section 5.2, we numerically verify the theoretical results regarding the order of the GIBC in the case of regular bounaries. Section 5.3 is devoted to boundaries with corner singularities. We numerically show how the GIBC accuracy can be deteriorated in this case. We also show how the combination between local mesh refinement and the use of GIBC restores the accuracy order.

\section{Two model problems}

Let $\Omega$ be an open bounded domain of $\mathbb{R}^{3}$ with connected complement and regular boundary $\Gamma:=\partial \Omega$, totally or partly filled by a penetrable material. We are interested in approximating the scattered field in $\mathbb{R}^{3} \backslash \bar{\Omega}$ in the situations where a boundary layer, whose width is small compared to the incident wavelength, is present near $\Gamma$. Two typical situations can be responsible of this boundary layer effect.

(a) Thin coating: in this case the penetrable region is a coating of small width $\delta$ (the domain $\Omega^{\delta}$, see Figure 1) bounded by a perfectly conducting material. The boundary layer here is then due to the smallness of the physical thickness of the penetrable region.

(b) Highly conducting materials: in this case the penetrable region is the whole domain $\Omega$ but due to the high conductivity $\sigma^{\delta}$, there is a rapid exponential decay of the wave inside $\Omega$. The boundary layer here is then due to the physical properties of the material, its width is proportional to $\delta:=1 / \sqrt{\mu \omega \sigma^{\delta}}$, where $\omega$ is the frequency of the incident wave and $\mu$ is the relative magnetic permeability.

In these two situations, the effect of the thin layer on the scattered wave outside $\Omega$ can be approximated by the use of a local boundary condition on $\Gamma$ (GIBC). If the error between exact and approximated solutions is $O\left(\delta^{k+1}\right)$ than the GIBC is called GIBC of order $k$.

Let us assume for simplicity that the exterior domain $\left(\mathbb{R}^{3} \backslash \bar{\Omega}\right)$ is homogeneous and that the time and space scales are chosen such that the intrinsic impedance is 1 in this medium. We also 
assume that the time dependence is harmonic with a frequency $\omega>0$, i.e. the electromagnetic field is of the form

$$
\mathbf{E}^{\delta}(x, t)=\operatorname{Re}\left\{E^{\delta}(x) \exp (i \omega t)\right\}, \quad \mathbf{H}^{\delta}(x, t)=\operatorname{Re}\left\{H^{\delta}(x) \exp (i \omega t)\right\} .
$$

Denoting by $\left(E_{e}^{\delta}, H_{e}^{\delta}\right)=\left(E^{\delta}, H^{\delta}\right)_{\mid \mathbb{R}^{3} \backslash \bar{\Omega}}$, then

$$
i \omega E_{e}^{\delta}-\operatorname{curl} H_{e}^{\delta}=0, \quad i \omega H_{e}^{\delta}+\operatorname{curl} E_{e}^{\delta}=0, \quad \text { in } \mathbb{R}^{3} \backslash \bar{\Omega},
$$

where this total field can be decomposed into the sum of an incident field ( $\left.E_{\text {inc }}, H_{\text {inc }}\right)$ and a scattered one $\left(E_{e, s}^{\delta}, H_{e, s}^{\delta}\right)$ that satisfies the Silver-Müller radiation condition:

$$
\lim _{|x| \rightarrow \infty} \hat{x} \times(E \times \hat{x})-H \times \hat{x}=0
$$

uniformly with respect to $\hat{x}:=x /|x|$. Let $n$ denotes a regular normal field on $\Gamma$. Then,

$$
E_{e}^{\delta} \times n=E_{i}^{\delta} \times n, \quad H_{e}^{\delta} \times n=H_{i}^{\delta} \times n \quad \text { on } \Gamma,
$$

where $\left(E_{i}^{\delta}, H_{i}^{\delta}\right)$ denotes the electromagnetic field inside $\Omega$. Depending on cases (a) and (b) the equations satisfied by this field are different. Let $\mu, \varepsilon$ and $\sigma$ respectively denote the relative magnetic permeability, the relative electric permittivity and the conductivity inside $\Omega^{\delta}$ in case (a) and $\Omega$ in case (b). In case (a) these quantities are assumed to be independent of $\delta$ and

$$
\begin{cases}(i \omega \varepsilon+\sigma) E_{i}^{\delta}-\operatorname{curl} H_{i}^{\delta}=0, & \text { in } \Omega^{\delta}, \\ i \omega \mu H_{i}^{\delta}+\operatorname{curl} E_{i}^{\delta}=0, & \text { in } \Omega^{\delta}, \\ E_{i}^{\delta} \times n=0 & \text { on } \Gamma^{\delta},\end{cases}
$$

whereas in case (b) the conductivity depends on $\delta$ and is set to $\sigma:=\sigma^{\delta}=1 /\left(\mu \omega \delta^{2}\right)$, and

$$
\begin{cases}\left(i \omega \varepsilon+\frac{1}{\mu \omega \delta^{2}}\right) E_{i}^{\delta}-\operatorname{curl} H_{i}^{\delta}=0, & \text { in } \Omega, \\ i \omega \mu H_{i}^{\delta}+\operatorname{curl} E_{i}^{\delta}=0, & \text { in } \Omega .\end{cases}
$$

\section{Expression of the GIBCs}

We denote by $\left(E_{e}^{\delta, k}, H_{e}^{\delta, k}\right)$ the desired approximation of order $O\left(\delta^{k+1}\right)$ of the exact electromagnetic field $\left(E_{e}^{\delta}, H_{e}^{\delta}\right)$. It satisfies the standard Maxwell equations in the exterior domain

$$
i \omega E_{e}^{\delta, k}-\operatorname{curl} H_{e}^{\delta, k}=0, \quad i \omega H_{e}^{\delta, k}+\operatorname{curl} E_{e}^{\delta, k}=0, \quad \text { in } \mathbb{R}^{3} \backslash \bar{\Omega},
$$

and is the sum of the incident field $\left(E_{\mathrm{inc}}, H_{\mathrm{inc}}\right)$ and a scattered one that satisfies the radiation condition (2). The interface conditions on $\Gamma$ and Maxwell equations inside $\Omega$ satisfied by the exact solution are replaced by a $G I B C$ of order $k$ on $\Gamma$ that can be written in the form

$$
E_{e}^{\delta, k} \times n+i(\mu \omega) \mathcal{D}^{\delta, k}\left(n \times\left(H_{e}^{\delta, k} \times n\right)\right)=0,
$$

where $n$ denotes the normal to $\Gamma$ directed to the interior of $\Omega$ of and where $\mathcal{D}^{\delta, k}$ is a local boundary operator acting on tangential vector fields on $\Gamma$. The order of this operator increases with the desired order of accuracy. We shall restrict ourselves to GIBCs leading to operators of order $\leq 2$. Before giving the expression of this operator for different values of $k$ (that can be interpreted as successive approximations of the $H$-to-E map for the Maxwell equations inside $\Omega$ ), we need to first introduce some notation related to surface operators on $\Gamma$.

We denote by $\nabla_{\Gamma}$ the surface gradient on $\Gamma$ and $-\operatorname{div}_{\Gamma}$ its adjoint with respect to $L^{2}(\Gamma)$ inner product. We then define the surface curl of a tangential vector $V$ and the surface vector curl of a scalar function $u$ defined on $\Gamma$ by

$$
\operatorname{curl}_{\Gamma} V:=\operatorname{div}_{\Gamma}(V \times n) \text { and } \operatorname{curl}_{\Gamma} u:=\left(\nabla_{\Gamma} u\right) \times n .
$$


The curvature tensor $\mathcal{C}$ is defined by $\mathcal{C}:=\nabla_{\Gamma} n$. We recall that $\mathcal{C}$ is symmetric and $\mathcal{C} n=0$. We denote by $c_{1}$ and $c_{2}$ the eigenvalues of $\mathcal{C}$ (namely the principal curvatures associated with tangential eigenvectors $\left.\tau_{1}, \tau_{2}\right)$; then $g:=c_{1} c_{2}$ and $h:=\frac{1}{2}\left(c_{1}+c_{2}\right)$ are respectively the Gaussian and mean curvatures of $\Gamma$. To these curvatures we associate

$$
\mathcal{H}=h I_{\Gamma} \text { and } \mathcal{G}=g I_{\Gamma},
$$

where $I_{\Gamma}\left(x_{\Gamma}\right)$ denotes the projection operator on the tangent plane to $\Gamma$ at $x_{\Gamma}$.

Expression of $\mathcal{D}^{\delta, k}$ in the case of thin coatings (a). In his case, setting $\tilde{\varepsilon}:=\varepsilon+\frac{\sigma}{i \omega}$, we have the following expressions for $k=0,1,2$, (see [12])

$$
\left\{\begin{array}{l}
\mathcal{D}^{\delta, 0}=0 \\
\mathcal{D}^{\delta, 1}=\delta\left(1-\frac{1}{(\tilde{\varepsilon} \mu) \omega^{2}} \operatorname{curl}_{\Gamma} \operatorname{curl}_{\Gamma}\right) \\
\mathcal{D}^{\delta, 2}=\delta\left((1-\delta(\mathcal{C}-\mathcal{H}))-\frac{1}{(\tilde{\varepsilon} \mu) \omega^{2}} \operatorname{curl}_{\Gamma}(1-\delta h) \operatorname{curl}_{\Gamma}\right) .
\end{array}\right.
$$

Expression of $\mathcal{D}^{\delta, k}$ in the case of high conductivity (b). In his case we have the following expressions for $k=0,1,2,3$, (see [15])

$$
\left\{\begin{array}{l}
\mathcal{D}^{\delta, 0}=0 \\
\mathcal{D}^{\delta, 1}=\left(\frac{\sqrt{2}}{2}-i \frac{\sqrt{2}}{2}\right) \delta \\
\mathcal{D}^{\delta, 2}=\mathcal{D}^{\delta, 1}+i \delta^{2}(\mathcal{C}-\mathcal{H}) \\
\mathcal{D}^{\delta, 3}=\mathcal{D}^{\delta, 2}-\left(\frac{\sqrt{2}}{4}+i \frac{\sqrt{2}}{4}\right) \delta^{3}\left(\mathcal{C}^{2}-\mathcal{H}^{2}+(\varepsilon \mu) \omega^{2}+\nabla_{\Gamma} \operatorname{div}_{\Gamma}+\operatorname{curl}_{\Gamma} \operatorname{curl}_{\Gamma}\right) .
\end{array}\right.
$$

As one can notice from comparing both cases, the limit problem is the same boundary condition for perfect conductors, however, the expression and nature of $\mathcal{D}^{\delta, k}$ greatly differ for higher order approximations. For instance, in the case of thin coatings, a surface wave operator appears starting from the first order. But it is only at the third order that one can see an effect of the wave propagation along the scatterer boundary in case of high absorption. Also in this case, one can see that the second order operator appearing in the expression of $\mathcal{D}^{\delta, 3}$ has no fixed sign, which causes difficulties in the mathematical study of the well-posedness of the approximate problem [15].

We refer to [15] for the mathematical justification of the order of these conditions in case (b). The error analysis in case (a) can be easily deduced by using similar arguments.

Let us finally mention that there are other possible GIBCs of order $k$, that differ from the ones given above only by $O\left(\delta^{k+1}\right)$ terms. Even if they theoretically lead to the same accuracy, their adaptivity to a given numerical solver may be different (see $[14,12,21])$. We presented here the expressions that are directly obtained from the procedure hereafter detailed. It turns out that they are also suitable to a finite element implementation as presented in section 5 .

\section{Formal construction of the approximations}

The construction of the approximations can be obtained from power series expansions in $\delta$ of the solutions inside $\Omega$, after a scaling with respect to the normal coordinate to $\Gamma$. More precisely, for a sufficiently small given positive constant $\bar{\nu}(\bar{\nu}=\delta$ in case (a)) we define

$$
\Omega^{\bar{\nu}}=\{x \in \Omega ; \operatorname{dist}(x, \partial \Omega)<\bar{\nu}\}
$$

and to any $x \in \Omega^{\bar{\nu}}$ we uniquely associate the parametric coordinates $\left(x_{\Gamma}, \nu\right) \in \Gamma \times(0, \bar{\nu})$ through

$$
x=x_{\Gamma}+\nu n, \quad x \in \Omega^{\bar{\nu}} .
$$


Then the exact solution has the following expansions:

$$
\mid \begin{array}{ll}
E_{e}^{\delta}(x)=E_{e}^{0}(x)+\delta E_{e}^{1}(x)+\delta^{2} E_{e}^{2}(x)+\cdots & \text { for } x \in \mathbb{R}^{3} \backslash \bar{\Omega} \\
H_{e}^{\delta}(x)=H_{e}^{0}(x)+\delta H_{e}^{1}(x)+\delta^{2} H_{e}^{2}(x)+\cdots & \text { for } x \in \mathbb{R}^{3} \backslash \bar{\Omega}
\end{array}
$$

where $E_{e}^{\ell}, H_{e}^{\ell}, \ell=0,1, \cdots$ are functions defined on $\mathbb{R}^{3} \backslash \bar{\Omega}$ and

$$
\mid \begin{array}{ll}
E_{i}^{\delta}(x)=E_{i}^{0}\left(x_{\Gamma}, \nu / \delta\right)+\delta E_{i}^{1}\left(x_{\Gamma}, \nu / \delta\right)+\delta^{2} E_{i}^{2}\left(x_{\Gamma}, \nu / \delta\right)+\cdots & \text { for } x \in \Omega^{\bar{\nu}} \\
H_{i}^{\delta}(x)=H_{i}^{0}\left(x_{\Gamma}, \nu / \delta\right)+\delta H_{i}^{1}\left(x_{\Gamma}, \nu / \delta\right)+\delta^{2} H_{i}^{2}\left(x_{\Gamma}, \nu / \delta\right)+\cdots & \text { for } x \in \Omega^{\bar{\nu}}
\end{array}
$$

where $x, x_{\Gamma}$ and $\nu$ are as in (10). In case (b), $E_{i}^{\ell}\left(x_{\Gamma}, \eta\right), H_{i}^{\ell}\left(x_{\Gamma}, \eta\right): \Gamma \times \mathbb{R}^{+} \mapsto \mathbb{C}$ and

$$
\mid \begin{array}{ll}
\lim _{\eta \rightarrow \infty} E_{i}^{\ell}\left(x_{\Gamma}, \eta\right)=0 & \text { for a.e. } x_{\Gamma} \in \Gamma, \\
\lim _{\eta \rightarrow \infty} H_{i}^{\ell}\left(x_{\Gamma}, \eta\right)=0 & \text { for a.e. } x_{\Gamma} \in \Gamma .
\end{array}
$$

These conditions ensure that $E_{i}^{\ell}$ and $H_{i}^{\ell}$ are exponentially decreasing inside the obstacle. In case (a) these functions are only defined for $\eta \leq 1$ and are such that

$$
E_{i}^{\ell}\left(x_{\Gamma}, 1\right) \times n=0 \text { for a.e. } x_{\Gamma} \in \Gamma,
$$

which is obtained from the perfect conductor condition on $\Gamma^{\delta}$. The set of equations satisfied by the terms of these expansions can be found from equating the terms of same order with respect $\delta$ after inserting them into the equations of the problem. This identification process is based on using the expression the curl operator in parametric coordinate. For instance, it is shown in $[12,15]$ that

$$
\begin{aligned}
\operatorname{curl} V= & {\left[\left(\frac{1}{j(\nu)}(I+\nu \mathcal{M}) \nabla_{\Gamma}\right) \cdot(\tilde{V} \times n)\right] n+\left[\frac{1}{j(\nu)}(I+\nu \mathcal{M}) \nabla_{\Gamma}(\tilde{V} \cdot n)\right] \times n } \\
& -\left[\frac{1}{j(\nu)}(\mathcal{C}+\nu \mathcal{G}) \tilde{V}\right] \times n-\partial_{\nu}(\tilde{V} \times n),
\end{aligned}
$$

for regular functions $V$ defined on $\Omega^{\bar{\nu}}$, where $\tilde{V}$ is defined on $\Gamma \times(0, \bar{\nu})$ by $\tilde{V}\left(x_{\Gamma}, \nu\right)=V\left(x_{\Gamma}+\nu n\right)$, and where the tangential operator $\mathcal{M}$ is defined on $\Gamma$ by $\mathcal{M C}=\mathcal{G}$, and $j(\nu):=\operatorname{det}(I+\nu \mathcal{C})=$ $1+2 \nu h+\nu^{2} g$.

The obtained set of equations enables an inductive characterization of the asymptotic expansion terms. In addition, analytic formulas of $E_{i}^{\ell}$ and $H_{i}^{\ell}$ in terms of $\eta$ and the boundary tangential values of $H_{e}^{k}$ on $\Gamma, k \leq \ell-1$, can be established (however this technical task becomes more and more involving as $k$ increases). These expressions are used in setting up the GIBCs.

Getting the GIBC expressions. The GIBC of order $k$ is obtained by considering the truncated expansion

$$
\tilde{E}^{\delta, k}:=\sum_{\ell=0}^{k} \delta^{\ell} E_{e}^{\ell} \quad \text { and } \quad \tilde{H}^{\delta, k}:=\sum_{\ell=0}^{k} \delta^{\ell} H_{e}^{\ell}
$$

as an approximation of order $k+1$ of respectively $E_{e}^{\delta}$ and $H_{e}^{\delta}$. Using the first interface condition in (3), one has

$$
\left.\tilde{E}^{\delta, k}\right|_{\Gamma}\left(x_{\Gamma}\right) \times n=\sum_{\ell=0}^{k} \delta^{\ell} E_{i}^{\ell}\left(x_{\Gamma}, 0\right) \times n \text { for } x_{\Gamma} \in \Gamma .
$$

Substituting the computed expressions of $E_{i}^{\ell}\left(x_{\Gamma}, 0\right)$ into (15) then leads to a boundary condition of the form

$$
\tilde{E}^{\delta, k} \times n+i(\mu \omega) \mathcal{D}^{\delta, k}\left(n \times\left(\tilde{H}^{\delta, k} \times n\right)\right)=\delta^{k+1} g_{k}^{\delta} \text { on } \Gamma
$$

where $\left\|g_{k}^{\delta}\right\|_{L_{t}^{2}(\Gamma)}$ is uniformly bounded with respect to $\delta$ and where $\mathcal{D}^{\delta, k}$ is some boundary operator (the one previously given for $k=0,1, \cdots)$. The GIBC of order $k$ that defines $E_{e}^{\delta, k}$ and $H_{e}^{\delta, k}$ is then obtained by neglecting the right-hand side of (16). 
Let us mention that once $\left(E_{e}^{\delta, k}, H_{e}^{\delta, k}\right)$ are computed, one can also get an approximation of order $O\left(\delta^{k+1}\right)$ of the field inside the medium through an analytic formula. This formula is a direct consequence of the analytic expressions of $E_{i}^{\ell}$ (see [15]).

\section{Numerical discussion in the case of high absorption}

We discuss in this section the numerical accuracy of the GIBCs and their validity for singular geometries. We shall restrict ourselves to the case (b) and to axi-symmetric obstacles which authorizes the use of the 3-D axisymmetric $H_{\text {curl }}$ spectral isoparametric $Q_{\ell}$ finite elements [10]. The numerical method uses a variational formulation and a Fourier expansion in the azimuthal variable as detailed in the next section. To simulate the radiation condition at infinity we use a coupling between finite element method and integral equation on the exterior surface (see [17]). For the discretization of this condition, we introduce a Lagrange multiplier (the surfacic current on the exterior surface), using the same idea that we explain in the next section for the treatment of the generalized impedance condition. A direct solver (LU factorization) is used for the numerical computations.

\subsection{Variational formulation and Fourier decomposition.}

In order to simplify the notation we shall simply denote by $(E, H)$ the electromagnetic field that satisfies (6-7). The used variational formulation is a classical $H_{\text {curl }}$ formulation for the electric field $E$. In order to take in account the GIBC on $\Gamma$, the magnetic current $J$ is introduced

$$
J:=n \times H \quad \text { on } \Gamma
$$

as an additional unknown on $\Gamma$. We then rewrite the GIBC for $E \times n$ and $J$ to complete the classical $H_{\text {curl }}$ formulation. The GIBCs of order 1 to 3 , can be synthetically written in the form

$$
E \times n+\beta n \times J+\gamma\left[\nabla_{\Gamma}\left(\operatorname{div}_{\Gamma}(n \times J)\right)-n \times \nabla_{\Gamma}\left(\operatorname{div}_{\Gamma}(J)\right]=0, \quad \text { on } \Gamma\right.
$$

where $\beta$ is a matrix and $\gamma$ is a constant. The unknown $J \in V(\Gamma)$ where $V(\Gamma)=L_{t}^{2}(\Gamma)$ in the cases where $\gamma=0$ (the subscript $\mathrm{t}$ referring to tangential functions) and $V(\Gamma)=H_{t}^{1}(\Gamma)$, the subspace of $L_{t}^{2}(\Gamma)$ fields with square integrable surfacic gradient, otherwise.

The variational formulation associated with (17) can be written in the form

$$
\mid \begin{aligned}
& \int_{\Gamma} E \times n \cdot \bar{\varphi} d \Gamma+\int_{\Gamma} \beta(n \times J) \cdot \bar{\varphi} d \Gamma \\
& \quad-\gamma \int_{\Gamma}\left[\operatorname{div}_{\Gamma}(n \times J) \operatorname{div}_{\Gamma}(\bar{\varphi})+\operatorname{div}_{\Gamma}(n \times \bar{\varphi}) \operatorname{div}_{\Gamma}(J)\right] d \Gamma=0, \quad \forall \varphi \in V(\Gamma) .
\end{aligned}
$$

In the case on an axi-symmetric obstacle $\Omega$, the resolution of the 3 -D problem can be reduced into the resolution of a series of 2 -D problems that we shall describe here after. Let $(r, \theta, z)$ be the cylindrical coordinate system, we assume that the boundary $\Gamma$ is generated by rotating with respect to the $z$ axis a curve $\Gamma_{g}$ of the plane $\theta=$ constant, parameterized by:

$$
M(\xi)=(r(\xi), z(\xi)) \quad \xi \in I \subset \mathbb{R} .
$$

Let $\Delta(\xi):=\sqrt{\left|r^{\prime}(\xi)\right|^{2}+\left|z^{\prime}(\xi)\right|^{2}}$ then, $d \Gamma=r(\xi) \Delta(\xi) d \theta d \xi=r(\xi) d \theta d s(\xi)$ where $d s(\xi):=\Delta(\xi) d \xi$ is the curvilinear measure along $\Gamma_{g}$. The tangent vectors $\tau_{1}(\xi)$ and $\tau_{2}(\xi)$ associated with principal curvatures of $\Gamma$ and the inward normal $n(\xi)$ form an orthonormal basis of $\mathbb{R}^{3}$ and are given by

$$
\tau_{1}(\xi)=\frac{1}{\Delta(\xi)}\left(\begin{array}{c}
r^{\prime}(\xi) \cos \theta \\
r^{\prime}(\xi) \sin \theta \\
z^{\prime}(\xi)
\end{array}\right) \quad \tau_{2}(\xi)=\left(\begin{array}{c}
-\sin \theta \\
\cos \theta \\
0
\end{array}\right) \quad n(\xi)=\frac{1}{\Delta(\xi)}\left(\begin{array}{c}
-z^{\prime}(\xi) \cos \theta \\
-z^{\prime}(\xi) \sin \theta \\
r^{\prime}(\xi)
\end{array}\right)
$$


The principal curvatures $c_{1}(\xi)$ and $c_{2}(\xi)$ are given by

$$
c_{1}(\xi)=\frac{\left(r^{\prime \prime} z^{\prime}-z^{\prime \prime} r^{\prime}\right)(\xi)}{\Delta(\xi)^{3}} \quad \text { and } \quad c_{2}=-\frac{z^{\prime}(\xi)}{r(\xi) \Delta(\xi)} .
$$

Let $\left(J_{1}(\xi, \theta), J_{2}(\xi, \theta)\right)$ such that $J(\xi, \theta)=J_{1} \tau_{1}+J_{2} \tau_{2}$, is a tangential vector field then

$$
\operatorname{div}_{\Gamma} J=\frac{1}{\Delta(\xi)} \frac{\partial J_{1}}{\partial \xi}+\frac{r^{\prime}(\xi)}{r(\xi) \Delta(\xi)} J_{1}+\frac{1}{r(\xi)} \frac{\partial J_{2}}{\partial \theta}=\frac{\partial J_{1}}{\partial s}+\frac{r^{\prime}(\xi)}{r(\xi) \Delta(\xi)} J_{1}+\frac{1}{r(\xi)} \frac{\partial J_{2}}{\partial \theta} .
$$

The axisymmetric configurations enables us to apply Fourier series with respect to the $\theta$ variable. More precisely, we seek the solution in the form

$$
E(r, \theta, z)=\sum_{m=-\infty}^{+\infty} \hat{E}_{m}(r, z) e^{-i m \theta} \quad J(\xi, \theta)=\sum_{m=-\infty}^{+\infty} \hat{J}_{m}(\xi) e^{-i m \theta} .
$$

For each $m$, the field $\left(E_{m}, J_{m}\right)$ satisfies a $2 \mathrm{D}$ problem in cylindrical coordinates $(r, z)$ where $E_{m}$ is sought in a 2D $H$ (curl)-like space ([10]) adapted to particular coordinates and $J_{m}$ is searched in $H^{1}\left(\Gamma_{g}\right)^{2}$ (we refer here to the case $k=3$ ). In particular, introducing the curvilinear operator

$$
\operatorname{div}_{s}^{m} J:=\frac{\partial J_{1}}{\partial s}+\frac{r^{\prime}}{r \Delta} J_{1}-\frac{i m}{r} J_{2}
$$

one easily sees that (18) results into

$$
\begin{aligned}
\int_{\Gamma_{g}}\left(\hat{E}_{m} \times n\right) \cdot \bar{v} r d s+\int_{\Gamma_{g}} \beta\left(n \times \hat{J}_{m}\right) \cdot \bar{v} r d s \\
\quad-\gamma \int_{\Gamma_{g}}\left(\operatorname{div}_{s}^{m}\left(n \times \hat{J}_{m}\right) \operatorname{div}_{s}^{m}(\bar{v})+\operatorname{div}_{s}^{m}(n \times \bar{v}) \operatorname{div}_{s}^{m}\left(\hat{J}_{m}\right)\right) r d s=0
\end{aligned}
$$

for all $v \in H^{1}\left(\Gamma_{g}\right)^{2}$. While the electric field is approximated with $H_{\text {curl }}$ spectral isoparametric $Q_{\ell}$ finite elements, the current density is discretized using standard isoparametric continuous $P_{\ell}$ finite elements. Let us mention that we get a good approximation of the curvature terms (hidden in the matrix $\beta$ ) as soon as $\ell \geq 2$.

\subsection{Validation of the GIBCs for smooth boundaries.}

In our numerical experiments, we compute an approximate solution $E^{\delta, k}$ ( $k$ refers to the order of the GIBC) with the method presented in section 5.1. The polynomial order $\ell=7$ of the finite element approximation is large enough and the computational mesh is fine enough so that the discretization errors can be considered as negligible. We compute a reference solution $E^{\delta}$ after having meshed the interior of the obstacle. The mesh is constructed in such a way that the boundary layer effect is correctly taken into account. The accuracy of the GIBC is tested by representing the error fiunctional

$$
\text { Error }:=\left\|\operatorname{curl} E^{\delta}-\operatorname{curl} E^{\delta, k}\right\|_{L^{2}(D \backslash \bar{\Omega})} /\left\|\operatorname{curl} E^{\delta}\right\|_{L^{2}(D \backslash \bar{\Omega})}
$$

in terms of $\delta$, where $D$ is our bounded domain of computations.

We choose a (non convex) peanut geometry as an example of $C^{1}$ domains: see Figure 3-left (in each picture, we shall superpose to the obstacle geometry the distribution of the current density modulus for a given scattering experiment). We compute the scattering of an incident plane wave propagating along the axis of revolution of the obstacle. In this case only the two harmonic modes $m=1$ and $m=-1$ (cf section 5.1 ) have to be computed. This wave propagates from top to bottom, according to Figure 3-left. In the first simulation a moderate frequency is used: $\omega=0.2 \pi$, which corresponds to a wavelength two times smaller than the height of the scatterer. As shown 
by the error curves in Figure 3-right, one gets a convergence rate that roughly corresponds to the theoretical $O\left(\delta^{k+1}\right)$ for a GIBC of order $k$. More precisely one gets $O\left(\delta^{3.8}\right)$ for $k=3, O\left(\delta^{2.9}\right)$ for $k=2$ and $O\left(\delta^{2.5}\right)$ for $k=1$. In the second example shown in Figure 4, we increased the frequency: $\omega=\pi$. In this case one observes that the GIBC of order 1 and order 2 give similar precision (till 3 digits): an improvement between $k=1$ and $k=2$ would be observed only with smaller values of $\delta$. However, the condition of order 3 improves significantly the precision. This is (more or less) expected since when the wavelength is very small as compared with the smallest radius of curvature of the surface, the corrections due to geometrical terms are not significant: the wave "locally sees" the obstacle as a flat boundary, for which the curvature is 0 (therefore the conditions of order 1 and order 2 are the same in this case). The improvement observed in the case of the third order GIBC is due to the surface wave operator.
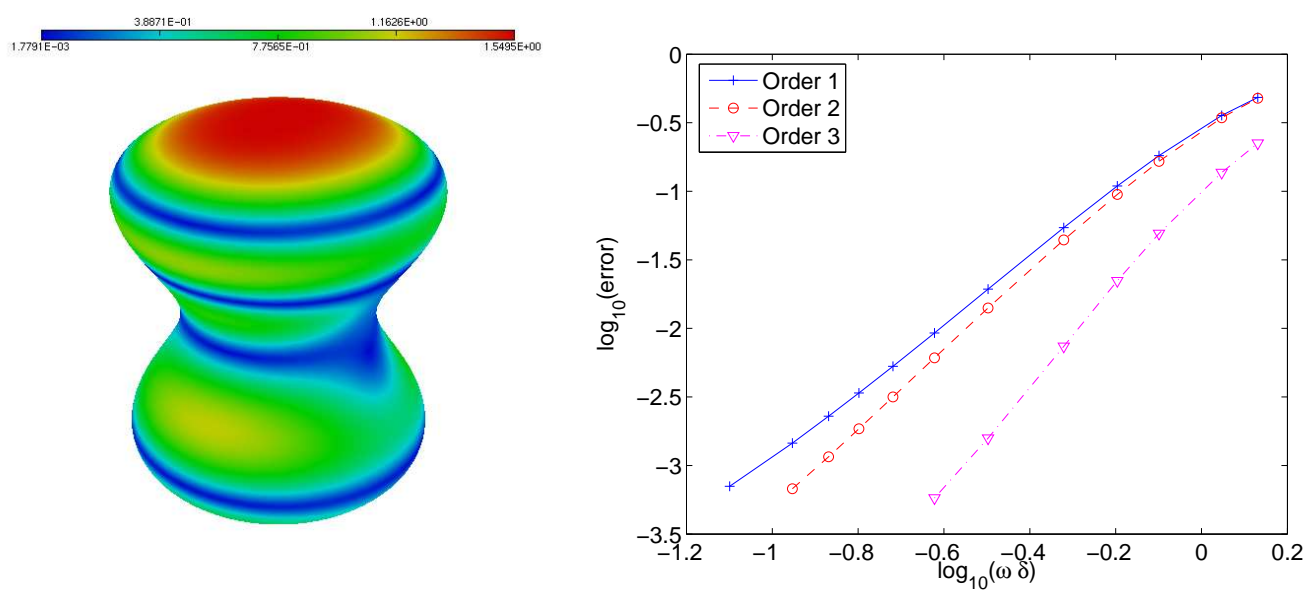

Figure 3: Left: $|\operatorname{Re}(J)|$ on the boundary $\Gamma$. Right: error curves in terms of $\delta$ (log-log scale).
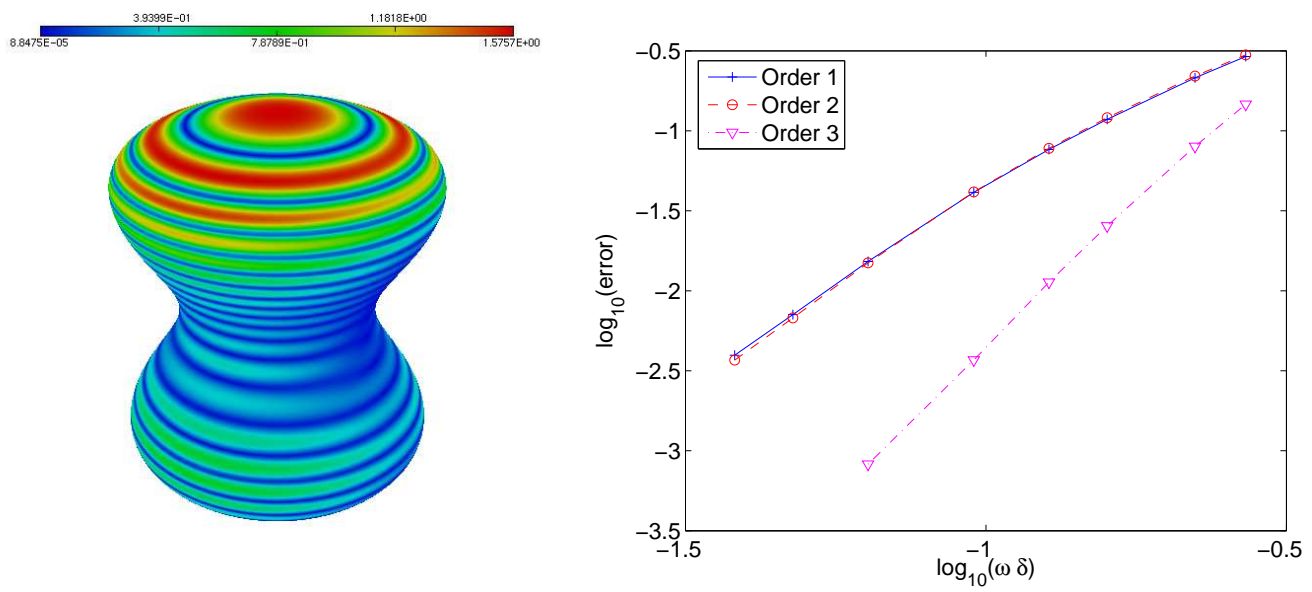

Figure 4: Left: $|\operatorname{Re}(J)|$ on the boundary $\Gamma$. Right: error curves in terms of $\delta$ (log-log scale).

\subsection{The treatment of singular boundaries.}

When the boundary is not smooth, the error analysis in [15] fails. One has even to be cautious in the definition of the GIBC of order 3 for non smooth boundaries [20]. To overcome this difficulty we propose to combine the use of local mesh refinement around the singularity and the use of GIBC in the region with regular boundary. The coupling between the two is done by introducing 
a fictitious regular boundary inside the absorbing medium at the singularity regions that links the regular parts of $\Gamma$ so that their union gives a $C^{1}$ surface $\tilde{\Gamma}$. The GIBC is then applied on $\tilde{\Gamma}$ and the small region around the singularity is treated as a part of the computational volume domain: see Figure 5-right as an example.

In the sequel, we shall compare the results obtained from a naive treatment of the singularity, consisting in applying (at the discrete level) the GIBC on $\Gamma$ as in the case of regular surfaces, with the results obtained after applying the numerical treatment explained above. The error curves associated to this treatment are labeled by adding the word "modified".
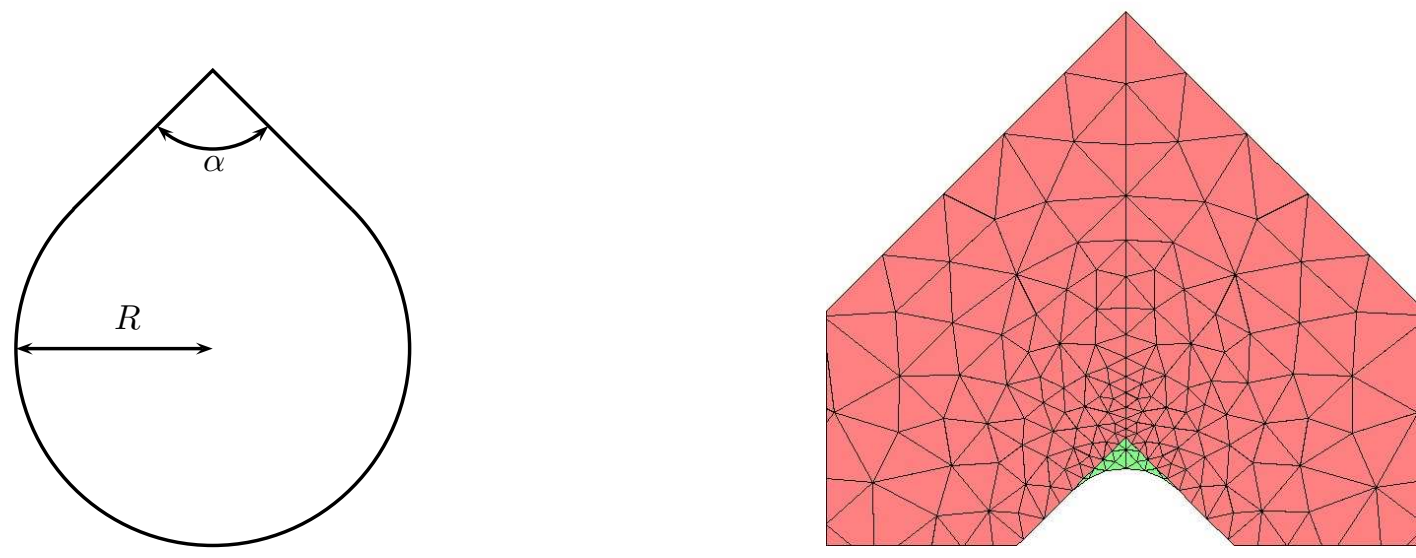

Figure 5: Left: the diedron-disk geometry. Right: example of a mesh including a small part (in green) of the absorbing medium.

The considered object is a "sharp ring" generated by rotating a diedron-disk around the $z$ axis (see Figure 6-left). The incident plane wave propagates as in section 5.2. In our first experiment we take $R=\pi$ and $\alpha=30$ degrees. The pulsation is chosen equal to $\omega=1$, so that the wavelength is two times smaller than the height of the scatterer. As shown by the error curves in Figure 6-right, the GIBC of order 1 or 3 fails to give the same convergence rate as in the case of smooth boundary. Let us notice, that both conditions give a convergence rate in $O\left(\delta^{0.9}\right)$, and no improvement is observed when the GIBC of order 3 is used instead of order 1 , for $\delta$ small enough. However, after applying the numerical treatment of the singularities, one significantly improves the accuracy: we get a convergence rate in $O\left(h^{2.7}\right)$ for the GIBC of order 1 , and a convergence rate in $O\left(\delta^{4.4}\right)$ for GIBC of order 3. 

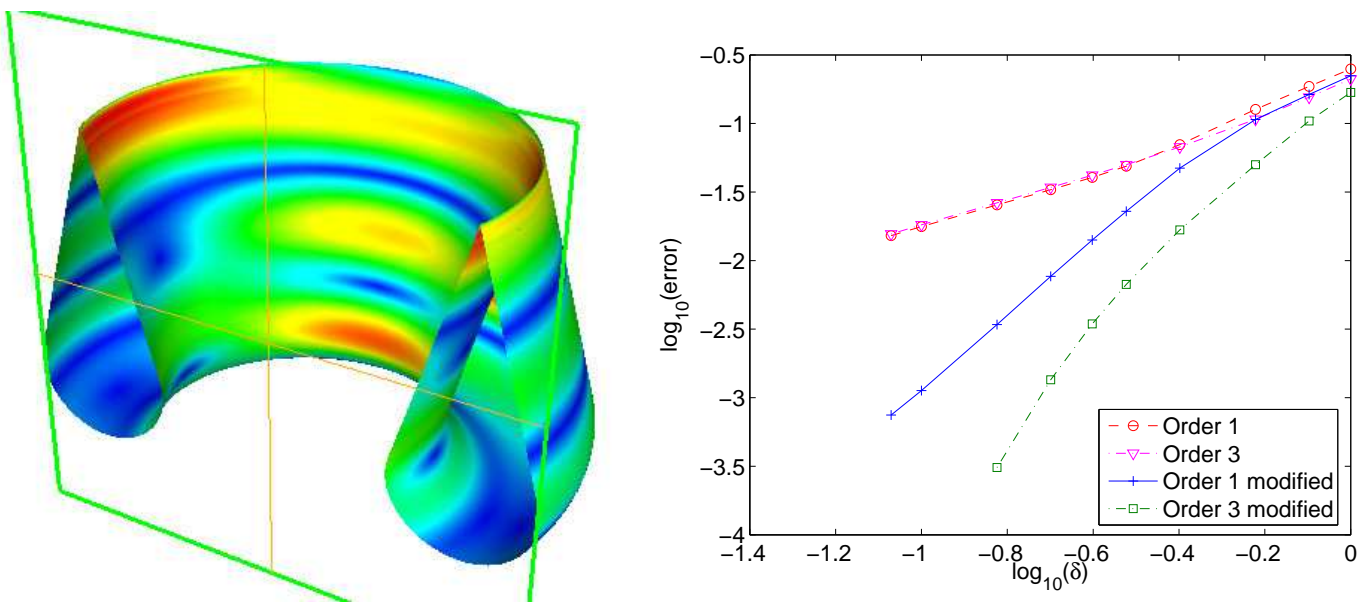

Figure 6: Left: $|\operatorname{Re}(J)|$ on the boundary $\Gamma$. Right: error curves in terms of $\delta$ (log-log scale).

Increasing the angle $\alpha$ reduces the singular behavior of the exact solution at the corner. Hence, for $\alpha=90$ degrees (see Figure 7-left), the standard GIBCs behaves slightly better that in the previous case (same convergence rate in $O\left(\delta^{1.2}\right)$ for the GIBCs), but their accuracy is still poor as compared with the case of regular geometries (especially for the third order one). Once again, the modified ones roughly restore the expected accuray (the measured convergence rate are respectively $O\left(\delta^{2.3}\right)$ $O\left(\delta^{2.7}\right)$ for orders 1 and 3$)$. Due to the weaker singularity of the obstacle, the improvement due to the treatment of the singularity is less spectacular than in our first experiment.
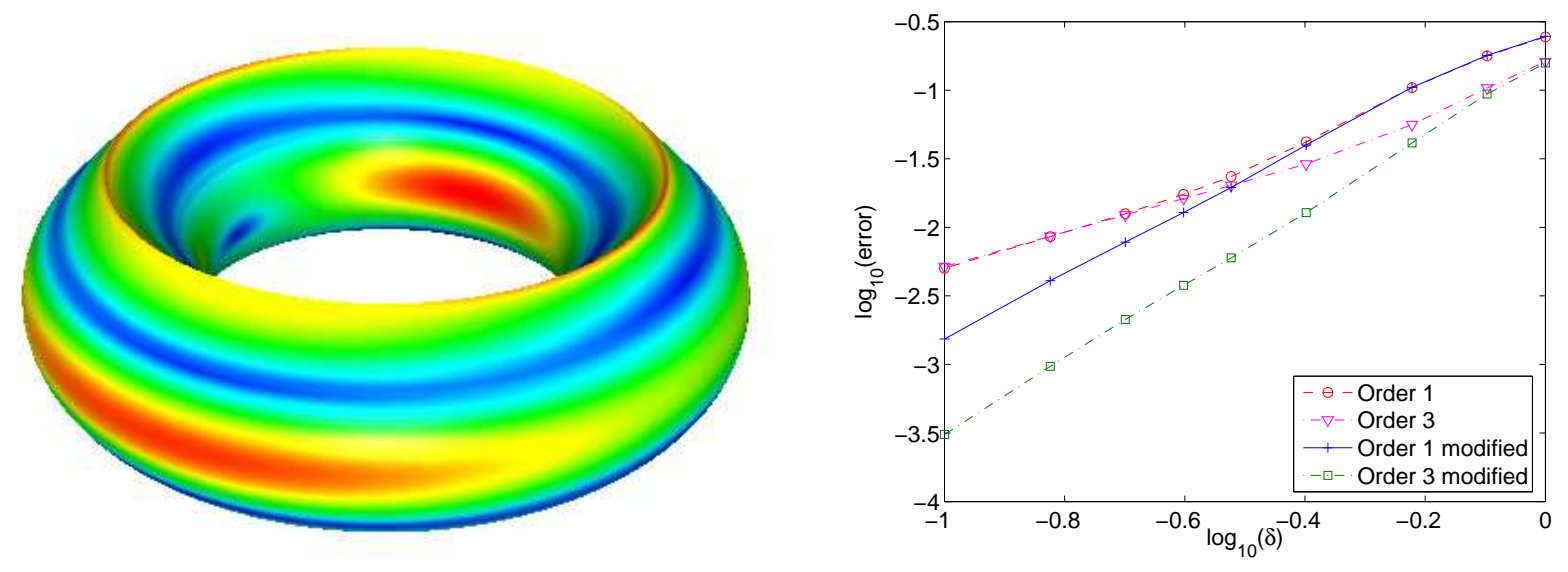

Figure 7: Left: $|\operatorname{Re}(J)|$ on the boundary $\Gamma$. Right: error curves in terms of $\delta$ (log-log scale).

However, when the frequency is increased, $\omega=2 \pi$, one recovers the same type of improvement than observed with the sharper geometry at lower frequency (see Figure 8). 

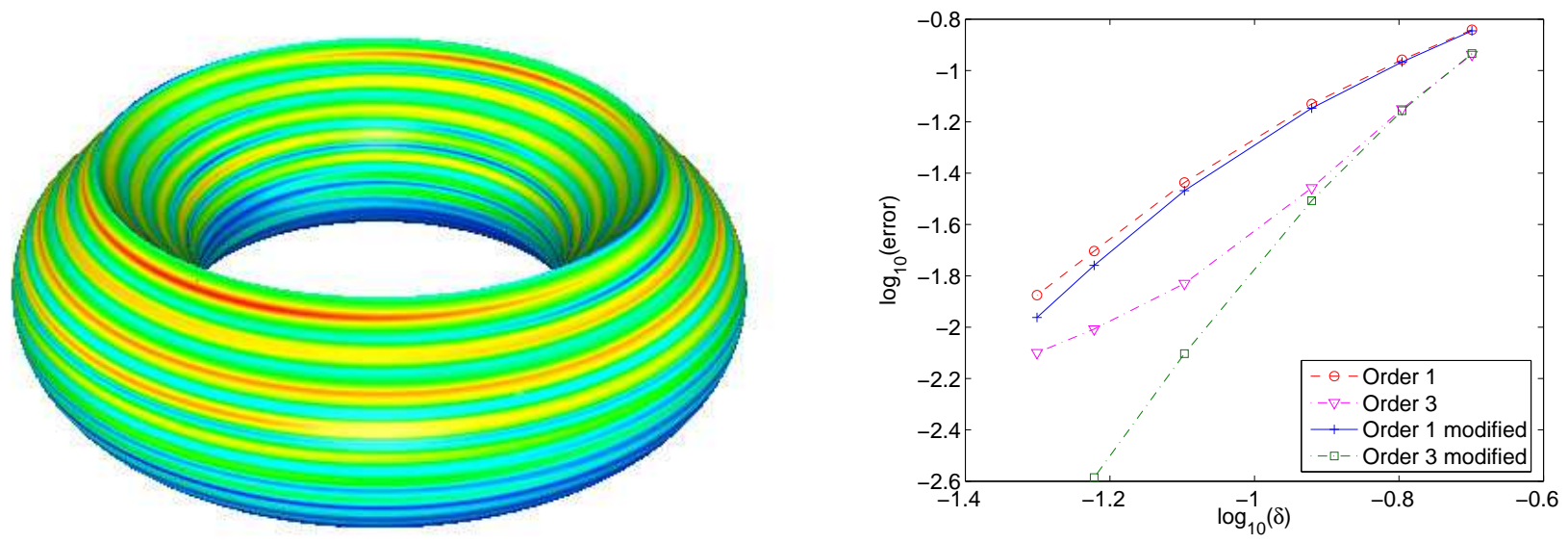

Figure 8: Left: $|\operatorname{Re}(J)|$ on the boundary $\Gamma$. Right: error curves in terms of $\delta$ (log-log scale).

\section{References}

[1] T. Abboud and H. Ammari, Diffraction at a curved grating. TM and TE cases, homogenization, J. Math. Anal and Appl., (1996), 202, 995-1026

[2] H. Ammari and C. Latiri-Grouz, Conditions aux limites approchés pour les couches minces périodiques en électromagnétisme, Math. Model. Numer. Anal., (1999), (33), 4,673-693,

[3] H. Ammari, C. Latiri-Grouz, and J.C. Nédélec, Scattering of Maxwell's equations with a Leontovich boundary condition in an inhomogeneous medium: a singular perturbation problem, SIAM J. Appl. Math. 59 (1999), no. 4, 1322-1334

[4] M. Artola and M. Cessenat, Scattering of an electromagnetic wave by a slender composite slab in contact with a thick perfect conductor. II. Inclusions (or coated material) with high conductivity and high permeability, C. R. Acad. Sci. Paris Sér. I Math. 313, no. 6, 381-385, (1991).

[5] M. Artola and M. Cessenat, The Leontovich conditions in electromagnetism. Les grands systèmes des sciences et de la technologie, RMA Res. Notes Appl. Math. 28, 11-21 Masson, Paris (1994).

[6] X. Antoine and H. Barucq, Microlocal diagonalization of strictly hyperbolic pseudodifferential systems and application to the design of radiation conditions in electromagnetism, SIAM J. Appl. Math. 61 (2001), no. 6, 1877-1905.

[7] X. Antoine, H. Barucq and L. Vernhet, High-frequency asymptotic analysis of a dissipative transmission problem resulting in generalized impedance boundary conditions, Asymptot. Anal. 26 (2001), no. 3-4, 257-283.

[8] A. Bendali and K. Lemrabet, The effect of a thin coating on the scattering of a time-harmonic wave for the Helmholtz equation, SIAM J. Appl. Math. 58 (1996), 1664-1693.

[9] N. Bartoli and A. Bendali, Robust and high-order effective boundary conditions for perfectly conducting scatterers coated by a thin dielectric layer. IMA J. Appl. Math. 67, no. 5, 479-508 (2002).

[10] M. Duruflé, Intégration numérique et éléments finis d'ordre élevé appliqués aux équations de Maxwell en régime harmonique, Thèse Université Paris IX-Dauphine (2006). 
[11] B. Engquist and J. C. Nédélec, Effective boundary conditions for acoustic and electromagnetic scattering in thin layers, Ecole Polytechnique-CMAP (France). 278 (1993).

[12] H. Haddar and P. Joly. Stability of thin layer approximation of electromagnetic waves scattering by linear and non linear coatings, J. Comp. and Appl. Math. 143, n. 2, pp 201-236, (2002).

[13] H. Haddar and P. Joly. Effective Boundary Conditions For Thin Ferromagnetic Layers; the One-Dimensional Model,

Siam J. Appl. Math. Vol. 61, No 4, pp 1386-1417, (2001).

[14] H. Haddar, P. Joly and H.M. Nguyen. Generalized Impedance Boundary Conditions for Scattering by Strongly Absorbing Obstacles: The Scalar Case, Math. Models and Meth. in Appl. Sci. Volume 15, n. 8, pp 1273-1300, (2005).

[15] H. Haddar, P. Joly and H.M. Nguyen. Generalized Impedance Boundary Conditions for Scattering by Strongly Absorbing Obstacles: The Maxwell Case, To appear (2005).

[16] O. Lafitte, Diffraction in the high frequency regime by a thin layer of dielectric material. I. The equivalent impedance boundary condition. SIAM J. Appl. Math. 59, no. 3, pp 1028-1052, (1999).

[17] V. Levillain, Couplage éléments finis-équations intégrales pour la résolution des équations de Maxwell en milieu hétérogène, Thèse école Polytechnique 1991.

[18] S.M. Rytov, Calcul du skin-effect par la méthode des perturbations, Journal de Physique USSR. 2, 233-242 (1940).

[19] T.B.A. Senior and J.L. Volakis, Approximate boundary conditions in electromagnetics, IEE Electromagnetic waves series (1995).

[20] E. M. Stein. Singular Integrals and Differentiability Properties of Functions, Princeton Mathematical Series, No. 30 , N.J. (1970)

[21] L. Vernhet, Boundary element solution of a scattering problem involving a generalized impedance boundary condition, Math. Methods Appl. Sci. 22 (1999), no. 7, 587-603. 